\title{
Glittering prizes
}

\section{Gold has many applications in nanotechnology and there are plenty more opportunities to explore.}

The Royal Bank Plaza building in Toronto's financial district has more than 14,000 windows, which is a lot of glass, and each of them is coated with a thin layer of gold, which is a lot of precious metal - the gold on the windows is worth over $\$ 1$ million according to estimates ${ }^{1}$. By reflecting heat and infrared radiation, the gold coating reduces heating and ventilation costs in the building. Although the use and importance of gold in the worlds of jewellery and finance are well known, the 79th element in the periodic table is also found in many less glamorous applications, as this example and a recent report from the World Gold Council makes clear ${ }^{2}$.

\section{Researchers are investigating the use of gold nanoparticles in cancer treatment.}

In many ways gold is a classic nanomaterial. It is chemically inert in its bulk form. Indeed, in the past gold was "often regarded as a rather boring element from a chemical perspective" according to one leading chemist ${ }^{3}$. However, gold nanoparticles are anything but inert, and their ability to catalyse a number of important chemical reactions has been the focus of much research over the past decade, although it remains to be seen if gold nanocatalysts can be transferred from the research laboratory to the world of industry ${ }^{4}$.

One of gold's main advantages for many applications is that it does not oxidize. It is also malleable and ductile, a good conductor of electricity, and is not toxic. One can read papers about research that takes advantage of some or all of these properties in most issues of any journal that covers nanotechnology. The source and drain contacts in experiments on nanoelectronic devices, for example, are often made from gold (or gold and titanium) that has been deposited with lithographic techniques. And when researchers observed molecular orbital gating in a single-molecule transistor for the first time last year, the device had started out as a gold nanowire coated with the molecules 5 . And gold is still capable of springing surprises. On page 218 of this issue, for example, Jun Lou and co-workers report that it is possible to form a weld between two gold nanowires by simply bringing them into contact with each other.

The World Gold Council report focuses on two main areas: the environment and health. Environmental applications include water purification, fuel cells, solar cells and improved catalysts (which would increase the efficiency of a wide range of industrial processes). In medicine, a large community of researchers is investigating the use of gold nanoparticles in cancer treatment: the basic aim here is to develop systems that can recognize diseased cells and tissues, thus reducing harmful side effects by ensuring that powerful cancer drugs are delivered to tumours and not to healthy tissue. There are also drugfree approaches that involve heating the nanoparticles with radiation so that they 'fry' the tumour. Early detection of the recurrence of prostate cancer after surgery is yet another application ${ }^{6}$.

Gold has been very good for nanotechnology and this looks set to continue.

\section{References}

1. http://en.wikipedia.org/wiki/Royal_Bank_Plaza

2. Gold For Good: Gold and Nanotechnology in the Age of Innovation

(World Gold Council \& Cientifica, 2010); available at <http://www.gold.org/>

3. Hutchings, G. Nature Chem. 1, 584 (2009).

4. Vaughan, O. Nature Nanotech. 5, 5-7 (2010).

5. Song, H. Nature 462, 1039-1043 (2009).

6. Thaxton, C. S. et al. Proc. Natl Acad. Sci. USA 106, 18437-18442 (2009).

\section{Mass appeal}

\section{Graphene could one day be involved in a new definition of the kilogram.}

To the left of gold in the periodic table are two elements - platinum and iridium that are just as interesting in many ways. Like gold, platinum is malleable and ductile and widely used as a catalyst, whereas iridium is hard and brittle and extremely resistant to corrosion. And in a vault in a metrology laboratory in Sèvres, a suburb of Paris, is a cylinder made of a platinumiridium alloy that has a mass of exactly one kilogram because, in the International System of Units (SI), the kilogram is defined as being equal to the mass of this International Prototype Kilogram.

There are seven base units in SI and the kilogram is the only one that is defined by an artefact, which is prone to change with time, rather than by a fundamental physical property, which should not change with time and can also be reproduced in other laboratories. This is why the international metrology community is looking into the possibility of redefining the kilogram in terms of the fundamental physical constants, such as the electron charge and Planck's constant.

One proposal for replacing the platinum-iridium cylinder in Sèvres requires extremely accurate methods for measuring resistance and voltage (which are not base units). The primary standard for resistance is based on the quantum Hall effect in a two-dimensional electron gas in a semiconductor heterostructure, but on page 186 of this issue Alexander Tzalenchuk and co-workers report that they can measure this effect in graphene with an accuracy that approaches that of the semiconductor method. Moreover, the graphene devices (complete with gold-titanium contacts) have the advantage that they require lower magnetic fields and can operate at higher temperatures. One of the more unusual features of graphene is that the charge carriers behave as relativistic particles that do not have mass. As is pointed out on page 171, it would be ironic if such particles were involved in a new definition of the kilogram. 